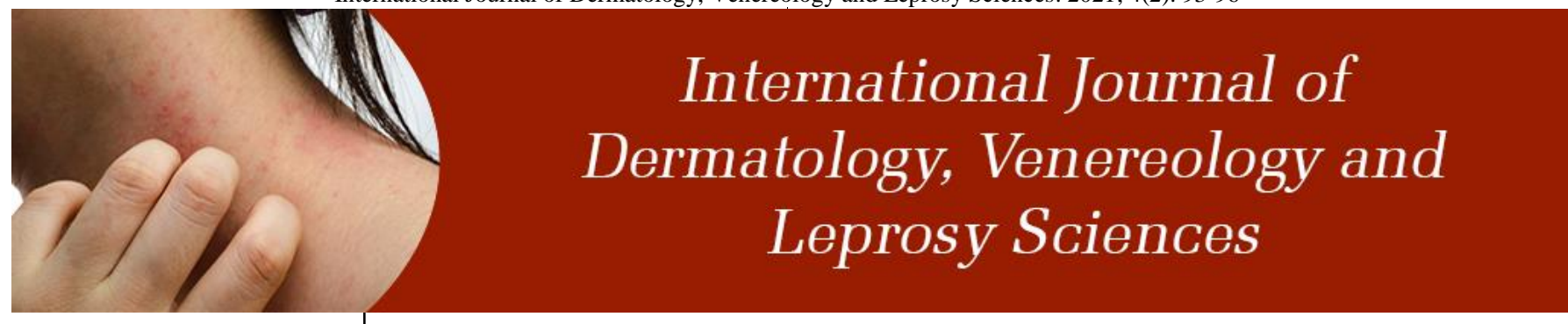

E-ISSN: 2664-942X

P-ISSN: 2664-9411

www.dermatologypaper.com

Derma 2021; 4(2): 93-96

Received: 29-07-2021

Accepted: 30-09-2021

Subadharshini B

Post Graduate, Department of Dermatology Venereology and Leprosy, Rajah Muthiah Medical College \& Hospital, Annamalai University, Chidambaram, Tamil Nadu, India

\section{Kaviarasan PK}

Head of the Department, Department of Dermatology Venereology and Leprosy, Rajah Muthiah Medical College \& Hospital, Annamalai University, Chidambaram

Tamil Nadu, India

\section{Kannambal K}

Associate Professor, Department of Dermatology Venereology and Leprosy, Rajah Muthiah Medical College \& Hospital, Annamalai University, Chidambaram,

Tamil Nadu, India

\section{Poorana B}

Assistant Professor, Department of Dermatology Venereology and Leprosy, Rajah Muthiah Medical College \& Hospital, Annamalai University, Chidambaram, Tamil Nadu, India

\section{Corresponding Author:}

Kaviarasan PK

Head of the Department, Department of Dermatology

Venereology and Leprosy, Rajah Muthiah Medical College \& Hospital, Annamalai University, Chidambaram, Tamil Nadu, India

\section{Role of biochip indirect immunofluorescence assay in diagnosis of autoimmune vesicobullous disorders in a tertiary care setting}

\author{
Subadharshini B, Kaviarasan PK, Kannambal K and Poorana B
}

DOI: https://doi.org/10.33545/26649411.2021.v4.i2a.102

\begin{abstract}
Introduction: Autoimmune vesicobullous disorders are rare life threatening conditions exclusively involving the skin and mucous membrane with heterogeneous symptoms and pose a major diagnostic challenge. It is important to rapidly diagnose vesiculobullous disorders to plan proper treatment. Older conventional methods of antibody detection are time consuming and can detect only specific antibodies in each test. BIOCHIP is a newer diagnostic tool which facilitates simultaneous, multiparametric analysis of all antibodies in a single miniature incubation field.

Aim: To evaluate the role of BIOCHIP mosaic based immunofluorescence test in the screening and diagnosis of autoimmune bullous disorsders.

Materials and Methods: Forty patients with autoimmune vesicobullous disorders were included in the study. Perilesional skin biopsy done for direct immunofluorescence and $5 \mathrm{ml}$ of blood sample was collected for BIOCHIP indirect immunofluorescence.

Results: Results of DIF and BIOCHIP were compared with clinical diagnosis. The sensitivity of DIF was $99 \%$ in all epidermal and subepidermal bullous diseases. The sensitivity of BIOCHIP in the diagnosis of pemphigus vulgaris, bullous pemphigoid, pemphigus foliaceus was almost $100 \%$ and comparable with that of DIF.

Conclusion: Thus the sensitivity and diagnostic accuracy of BIOCHIP is comparable with DIF making it a more effective practical screening tool for patients with suspected AIBD. Thus, BIOCHIP can be used as first line tool in the diagnosis of autoimmune bullous disorders.
\end{abstract}

Keywords: Vesicobullous, BIOCHIP mosaic, direct immunofluorescence, indirect immunofluorescence

\section{Introduction}

Autoimmune bullous disorders (AIBD) are characterized by autoantibodies against desmosomal proteins (in pemphigus), adhesion molecules of the dermal-epidermal junction (in pemphigoid diseases), and epidermal/ tissue transglutaminase (in dermatitis herpetiformis), respectively ${ }^{[1]}$.

Autoimmune vesicobullous diseases are infrequent and present with diverse symptoms. These diseases often pose a major diagnostic difficulty. The accurate and rapid establishment of diagnosis is inevitable to plan treatment and assess the prognosis. Though the conventional methods like direct immunofluorescence and indirect immunofluorescence have a very high sensitivity and specificity, they have their own limitations. DIF is considered the gold standard in diagnosis of these disorders, but the results are highly dependent on age of the lesion, sampling techniques, proper transport of the sample in transport medium, difficulty in performing oral and genital mucosal biopsy.

The newer BIOCHIP mosaic combines screening of target antigen and specific substrate in a single miniature incubation field. It facilitates simultaneous, multiparametric analysis of all relevant antibodies. A non-invasive technique like BIOCHIP with faster results as early as 100 minutes will ease the diagnosis and also helps in institution of specific therapy at the earliest ${ }^{[2]}$.

\section{Aims and Objectives}

To assess the role of BIOCHIP mosaic based immunofluorescence test in the screening and diagnosis of autoimmune bullous disorders. 


\section{Materials and Methods}

The current study was a prospective cross sectional study, conducted in the department of dermatology in a tertiary care centre, Tamilnadu. The study was done between October 2019 to September 2021 after obtaining institutional ethical committee clearance. Forty patients with autoimmune vesicobullous disorders satisfying inclusion criteria were included in the study. Peri-lesional skin biopsy from uninvolved normal skin was done for direct immunofluorescence. Five $\mathrm{ml}$ of blood sample was collected and serum was separated to perform BIOCHIP immunofluorescence test.

According to manufacturer instruction, substrates were incubated with diluted serum samples. Then the antibodies were stained with fluorescein-labelled anti-human antibodies. Fluorescence microscopy was used to evaluate the results ${ }^{[3]}$. The results of DIF and BIOCHIP assay were finally compared.

For the purposes of this study a diagnosis of pemphigus vulgaris was made when there was reactivity with DSG3, or both DSG3 and DSG1, and intercellular substance staining on monkey oesophagus was visualised. A diagnosis of pemphigus foliaceous was made when there was reactivity of DSG1, no reactivity of DSG3, and intercellular substance staining with monkey oesophagus. Bullous pemphigoid was diagnosed based on basement membrane zone staining on monkey oesophagus and or salt split skin reactivity on the epidermal roof side and or reactivity with BP180 and or BP230.

\section{Inclusion Criteria}

- All new cases of AIBD with skin/ skin and mucosal lesions irrespective of age and sex.

- Patients who have given consent and willing for follow up.

\section{Exclusion Criteria}

- Patients in clinical remission.

- Patients who are not willing to undergo the study, photography.

\section{Biochip Mosaic}

Dermatology Mosaic 7 BIOCHIP from EUROIMMUN, Germany was used in this study. The BIOCHIP method combines the screening of several autoantibodies and target antigen- specific substrates in a single miniature incubation field. This helps in simultaneous processing of various autoimmune bullous disease causing autoantibodies from suspected patients serum as a one-step procedure.

A standard BIOCHIP slide has 10 incubation fields with 6 different substrates. The various substrates include (i) frozen tissue section of monkey oesophagus (ii) $1 \mathrm{M} \mathrm{NaCl}$-split skin (iii) human embryonic kidney (HEK293) cells transfected with Dsg1 protein ectodomain (iv) HEK293 cells transfected with Dsg3 protein ectodomain (v) microdrops of
BP180 free antigen, and (vi) HEK293 cells transfected with C-terminal globular domain of the BP230 domain ${ }^{[4]}$.

A thin glass slide contains these coated substrates. Hence it enables us to screen 10 patients sera simultaneously for antibodies like BP180, BP230, DSG1 and DSG3 proving it to be a more efficient and cost saving method ${ }^{[4]}$.

Monkey esophagus sections reveal intercellular staining in the presence of pemphigus antibodies which are directed against the desmosomes. Presence of basement membrane zone staining in salt-split skin sections indicates presence of epidermal basement membrane zone autoantibodies. A linear fluorescent pattern in the basement membrane zone on the epidermal side is suggestive of bullous pemphigoid and on the dermal side is suggestive of epidermolysis bullosa acquisita (EBA). Granular fluorescence on sections coated with DSG1, DSG3, BP 180, BP 230 aids in diagnosis of pemphigus foliaceus, pemphigus vulgaris and bullous pemphigoid respectively ${ }^{[5]}$.

\section{Results}

This study included a total of 40 patients. Among them a clinical diagnosis of pemphigus foliaceus was made in 10 patients, pemphigus vulgaris in 9 patients, bullous pemphigoid in 10 patients, LIGA/CBDC in 4 patients and the diagnosis was inconclusive in 7 patients. With histopathological examination and DIF the diagnosis of pemphigus vulgaris in 11 patients and bullous pemphigoid in all patients were confirmed. Then BIOCHIP mosaic was done with sera from these patients. The BIOCHIP result was interpreted in six different substrates.

In pemphigus group $(n=11$ patients $)$ intercellular staining pattern was observed in all 11 patients. DSG 1 showed positive fluorescence in 8 patients and DSG 3 in 11 patients. Similarly in bullous pemphigoid group ( $\mathrm{n}=12$ patients) linear BMZ pattern was seen in all 12 patients. BP 180 was positive in 11 patients and BP 230 was positive in 8 patients. Results showed sensitivity of $73 \%$ for DSG 1, $100 \%$ for DSG 3, $78.5 \%$ for BP 180 and $57 \%$ for BP 230.

Also in comparison of BIOCHIP mosaic with DIF, the results showed a concordance of $100 \%$ in the diagnosis of pemphigus vulgaris $(n=11)$. In case of pemphigoid group what DIF diagnosed as bullous pemphigoid $(\mathrm{n}=12)$, biochip showed the diagnosis of bullous pemphigoid only in 11 patients and 1 patient showed a floor pattern in salt split skin. This patient did not show positivity for any of the target antigens and revealed a linear BMZ pattern in primate oesophagus and floor pattern in salt split skin. This patient could be any of the floor pattern staining diseases like EBA, anti laminin 332 pemphigoid or anti P-200 pemphigoid, which needs further evaluation with immunoblotting.

The correlation between BIOCHIP mosaic and DIF in the diagnosis of AIBD was assessed using Spearman's correlation coefficient and it was found to be statistically significant.

Table 1: Distribution of study population by clinical diagnosis, histopathological examination, direct immunofluorescence and indirect immunofluorescence

\begin{tabular}{|c|c|c|c|c|c|}
\hline & \multirow{2}{*}{ Clinical Diagnosis } & \multirow{2}{*}{ HPE } & \multicolumn{2}{|c|}{ DIF } & \multirow{2}{*}{ Biochip } \\
\hline & & & ICS & BMZ & \\
\hline $\mathrm{PF}$ & 10 & 10 & 10 & - & 10 \\
\hline PV & 9 & 10 & 9 & - & 11 \\
\hline BP & 10 & 12 & - & 10 & 14 \\
\hline LIGA/CBDC & 4 & 2 & - & 4 & 4 \\
\hline Inconclusive & 7 & 6 & 3 & 4(LIGA) & 1(NEGATIVE) \\
\hline Total & 40 & 40 & & 40 & 40 \\
\hline
\end{tabular}


However a diagnosis of linear IgA bullous dermatoses could not be made with BIOCHIP alone. Linear IgA deposits were demonstrated along the dermoepidermal junction in direct immunofluorescence. On doing BIOCHIP these patients sera showed roof pattern staining in salt split skin and was negative for BP180, BP 230.

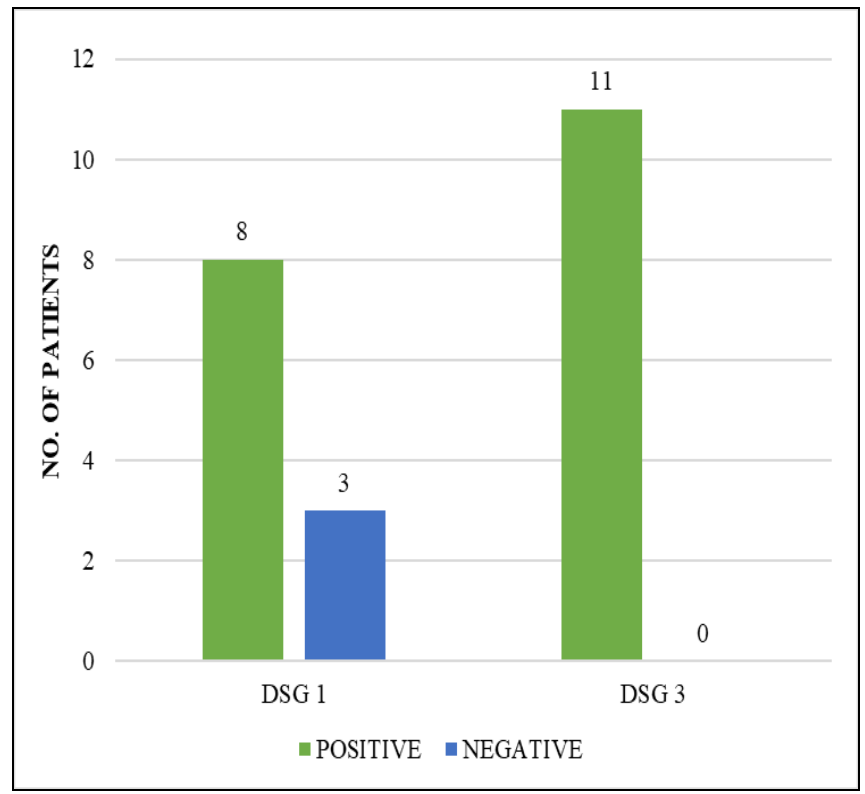

Fig 1: Distribution of pemphigus vulgaris group by desmoglein 1 $\&$ desmoglein 3 positivity

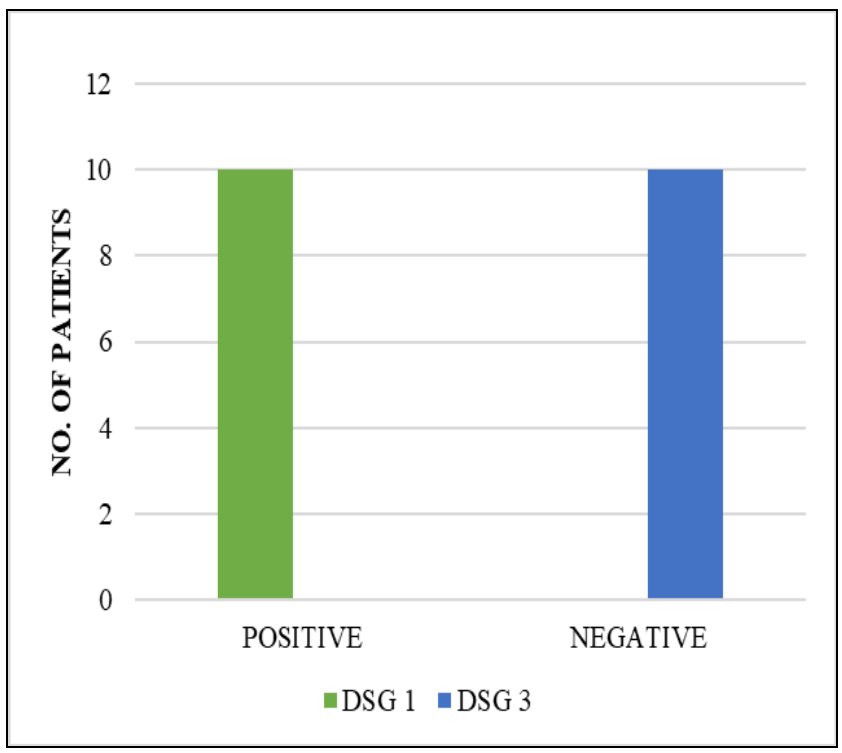

Fig 2: Distribution of pemphigus foliaceus by desmoglein $1 \& 3$ positivity

In patients with pemphigus vulgaris $(n=11)$, Desmoglein 1 was positive in 8 patients $(73 \%)$ and Desmoglein 3 was positive in all 11 patients $(100 \%)$.

In patients with pemphigus foliaceus, DSG 1 was positive in all 11 patients, DSG 3 was negative in all patients and they also revealed intercellular staining pattern in primate esophagus.

\section{BP 180 - PEMPHIGOID GROUP}

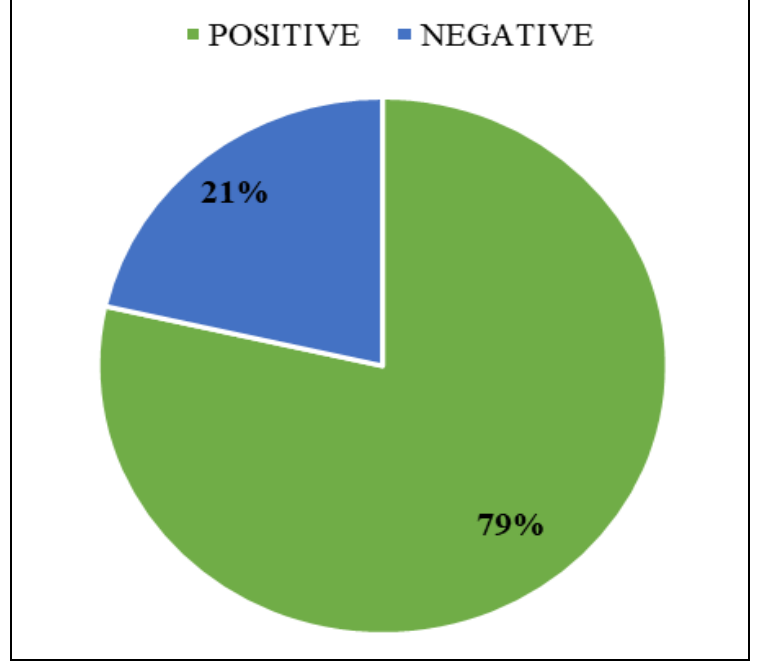

Fig 3: Distribution of pemphigoid group by BP 180 positivity

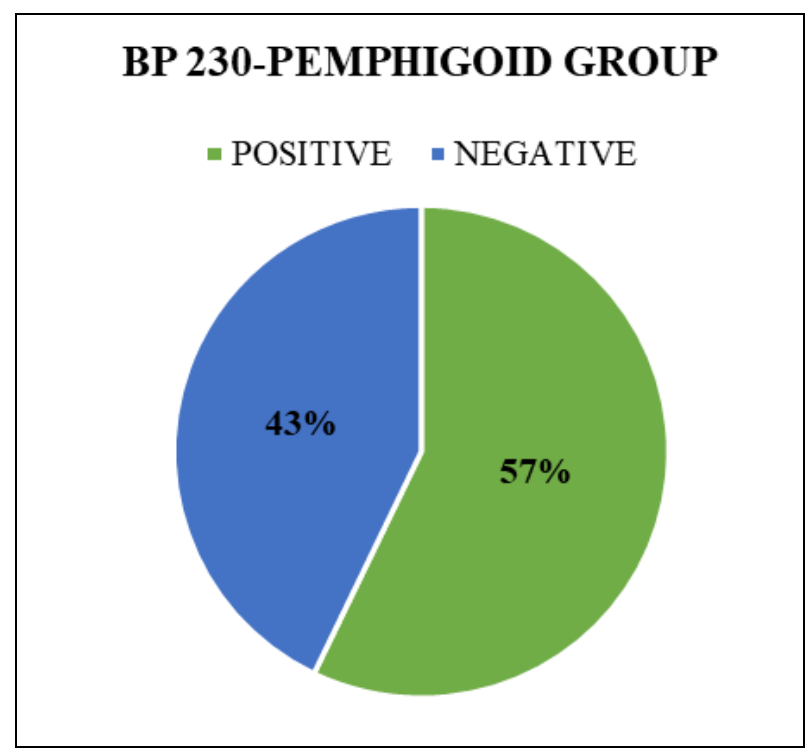

Fig 4: Distribution of pemphigoid group by BP 230 positivity

In patients with bullous pemphigoid $(n=14)$, BP 180 was positive in 11 patients $(79 \%)$ and negative in 3 patients $(21 \%)$. In patients with bullous pemphigoid $(n=14)$, BP 230 was positive in 8 patients $(43 \%)$ and negative in 3 patients $(57 \%)$.

\section{Discussion}

The aim of this study was to evaluate the performance of BIOCHIP mosaic in comparison to DIF. The BIOCHIP technique stands out to be a specific and sensitive diagnostic alternative to DIF in the diagnosis of autoimmune vesicobullous disorders ${ }^{[6]}$.

The BIOCHIP mosaic containing different tissue sections, cell substrates and antigenic molecules requires only a simple antibody incubation to obtain a detailed profile. It enables to search for different antibodies simultaneously ${ }^{[2]}$. It has various advantages like the capability to analyse several serum samples simultaneously, faster results (about 100 minutes), ready to use reagents in a convenient kit, easy visual interpretation of results which can be done even in small laboratories, the test can be done at room temperature and has low reagent consumption ${ }^{[2]}$. 
However, it has limitations in categorisation of pemphigoid diseases due to restricted antibody coating. This could be overcome by testing additional target antigens or immunoblotting. Thus, this new immunoassay can be used as an excellent screening test for patients with suspected autoimmune bullous skin disease, preserving the more expensive ELISA test in doubtful cases.

Van Beek et al. from Germany were the first to evaluate the utility of BIOCHIP mosaic in the diagnosis of AIBD. They compared BIOCHIP with the multistep algorithm described by Schimdt and Zillikens. The finally concluded that the BIOCHIP mosaic has a diagnostic efficacy comparable with other conventional multistep procedures ${ }^{[7]}$.

Tampoia et al. investigated the value of the BIOCHIP method by comparing it with two commercially available ELISA tests (MBL, Japan and EuroImmun, Germany) in the diagnosis of pemphigus and pemphigoid.

Later Russo et al. from Italy and Özkesici et al. from Turkey evaluated the utility of BIOCHIP in the diagnosis of AIBD by comparing it with ELISA. They concluded that the BIOCHIP method has a diagnostic accuracy comparable to ELISA ${ }^{[5]}$. However in our study comparison with ELISA was not done.

Thirumalae et al. study was done over 4 years with 209 samples BIOCHIP showed $92 \%$ positivity for pemphigus and $84 \%$ for pemphigoid groups. Indirect immunofluorescence by BIOCHIP method showed a good concordance with DIF ${ }^{[8]}$.

Arun Prasath et al compared the efficacy of BIOCHIP mosaic based immunofluorescence and direct immunofluorescence. BIOCHIP mosaic results showed a concordance of $100 \%$ in the diagnosis of pemphigus with DIF. Salt split skin substrate showed a roof pattern in 18 patients and floor pattern in 4 patients ${ }^{[6]}$. Similarly in our study 1 patient in pemphigoid group showed floor pattern staining without reactivity for other autoantibodies. This could be a case of floor pattern staining diseases like EBA, anti laminin 332 pemphigoid or anti P-200 pemphigoid, which needs further evaluation with immunoblotting.

Similar to the previous studies, the current study also revealed that Dsg 3 and BP 180 were the commonly detected antigens using BIOCHIP in pemphigus and pemphigoid groups, respectively. The pemphigus group showed high correlation which can be attributed to the fact that only two main target antigens are present in pemphigus group,both of which are also present in the BIOCHIP substrates. Whereas in the pemphigoid group other antigens in the basement membrane zone may be targeted in addition to BP 180 and BP 230 and this needs futhur evaluation with immunoblotting ${ }^{[6]}$.

\section{Limitations}

Lack of control group, small sample size.

\section{Conclusion}

Thus the novel BIOCHIP is a flexible, sensitive and specific alternative to detect autoantibodies in autoimmune vesicobullous disorders. It also helps in serological diagnosis of other autoimmune diseases by additional parameters. It is a complementary diagnostic tool due to its low cost, high sensitivity and easy execution.

\section{References}

1. Van Beek N, Rentzsch K, Probst C, Komorowski L, Kasperkiewicz M, Fechner $\mathrm{K}$ et al. Serological diagnosis of autoimmune bullous skin diseases: prospective comparison of the BIOCHIP mosaic-based indirect immunofluorescence technique with the conventional multi-step single test strategy. Orphanet J Rare Dis. 2012.

2. Zarian H, Saponeri A, Michelotto A, Zattra E, BelloniFortina A, Alaibac M. Biochip technology for the serological diagnosis of bullous pemphigoid. ISRN Dermatol. 2012;2012:237802.

3. Simpson K, Scardamaglia L, Kok Y, Vu M, Kidd D, Yap $\mathrm{T}$ et al. Comparison of the EUROIMMUN Dermatology Profile ELISA to the novel BIOCHIP Mosaic 7 for the diagnosis of immunobullous skin disease. Australas J Dermatol. 2021;62(3):314-322.

4. Yang A, Xuan R, Murrell DF. A new indirect immunofluorescence BIOCHIP method for the serological diagnosis of bullous pemphigoid: A review of literature. Australas J Dermatol. 2019;60(3):e173e177.

5. Russo I, Saponeri A, Peserico A, Alaibac M. The use of biochip immunofluorescence microscopy for the diagnosis of pemphigus vulgaris. Acta Histochem. 2014;116(5):713-6.

6. Arunprasath P, Rai R, Venkataswamy C. Comparative analysis of BIOCHIP mosaic-based indirect immunofluorescence with direct immunofluorescence in diagnosis of autoimmune bullous diseases: A cross sectional study. Indian Dermatol Online J 2020;11:9159.

7. Tampoia M, Zucano A, Villalta D, Antico A, Bizzaro N. Anti-skin specific autoantibodies detected by a new immunofluorescence multiplex biochip method in patients with autoimmune bullous diseases. Dermatology. 2012;225:37-44.

8. Tirumalae R, Kalegowda IY. Role of BIOCHIP Indirect Immunofluorescence Test in Cutaneous Vesiculobullous Diseases. Am J Dermatopathol. 2020;42(5):322-328. 